\title{
The Influence of the FFA on the Cognitive Dissonance of the Meat Paradox
}

\author{
Rachel Howard ${ }^{1}$ and Jennifer Poole ${ }^{1}$ \\ ${ }^{1}$ Walter L. Sickle High School, Tampa, FL, USA
}

\section{ABSTRACT}

The context of meat is an apt domain in which to explore the critical issues of preference and values (Rozin, 1996). This makes meat a significant interest in the psychological field as it is a key model of cognitive dissonance. This anomaly comprising of the connection between meat and ambivalence can be better known as the meat paradox. While there are many studies in the scholarly field about the morality and ethics associated with meat consumption (Loughnan et al., 2010), there is a lack of research about the meat paradox in individuals whose daily routines involve working and handling animals. In veterinary and agriculture-related classes, it is obvious that there is a wide range of perceptions about the ethics of consuming specific types of meat. The spectrum ranges from individuals who eat any type of meat to those who believe only farm-raised meat is morally acceptable, and finally, to those who believe that it is unethical to kill all animals for the primary purpose of food consumption. While it is not yet clear which kind of ethical belief is most abundantly held by the individuals, it is apparent that many people who are more willing to eat meat are in the FFA, an agricultural education organization. The understanding of a possible interaction of the FFA and ethical beliefs towards meat can inform psychologists of a solution to the meat paradox and provide more knowledge on what affects the change in ambivalence. The overall premise of this research project is to demonstrate a possible correlation between an animal-oriented organization, the FFA, and the cognitive dissonance of the meat paradox. These studies may suggest for further research into

\section{Literature Review}

\section{The Meat Paradox}

The meat industry, while vast in its comprehension, has several mental implications that affect the everyday consumer. Meat consumption's repercussions have increasingly affected the majority of the world's population, with Americans alone eating an alarming 264lbs of meat annually (Loughnan et al., 2014). The incorporation of meat into one's diet involves the psychological dissonance about the suffering of animals in conjunction with the inescapable actions of eating them. If the average individual perceives this paradox as noticeable, they will have to cope accordingly to these inconsistencies by reducing them, therefore refraining from discomfort (Buttlar and Walther, 2019). Loughnan and Buttlar and Walther's research are all relevant to this study as they discuss the meat industry and paradox in detail, in two reliable journals. They all, while differentiating between physical and emotional perspectives, agree that the meat industry is having a rising negative effect on consumers' mentalities. With meat increasing in inclusion into one's diet and the industry itself affecting customers' mentalities, it can consequently be inferred that the meat industry is having an expandingly harmful impact on its audience of consumers by questioning their beliefs confusing their reasons for eating meat. 


\section{FFA}

The FFA, previously standing for "Future Farmers of America" but now having a broader audience, is a national organization that strives to impart academic and personal growth to members through agricultural education and leadership skill opportunities. Regardless of the pursuance of a future career relating to agriculture, the organization believes in "growing leaders and building communities" (FFA, n.d.). There are several ways the FFA tries to achieve this goal, some of which include career development events, supervised agricultural experiences, community service opportunities, and in-school activities. While there is a stigma that it is associated with just agriculture and animals, they actually have a vaguer prospect, including aspects of varying interests such as floriculture, public speaking, aquaculture, forestry, landscape, and so forth. They hope to provide the next generation of leaders a successful future by endowing a positive difference in their lives. So far, they have accomplished this goal, and aim to continue this success through their motto of "Learning to Do, Doing to Learn, Earning to Live, Living to Serve" ("FFA Vision", n.d.).

\section{Raising Animals}

While there are a variety of interests that are related to the organization, a specific focus of some members is animals. Members select, raise, and show livestock through a Supervised Agricultural Experience project, referred to as a SAE. These types of SAEs allow students to gain real-world, hands-on experience within their chosen career path by enhancing their agricultural industry knowledge and gaining financial independence and management skills ("SAE", n.d.). The SAE for All organization is a relevant organization to this study as it provides promptly updated accurate information on the projects. Both the SAE For All organization and the FFA organization, while differing in their distinct beliefs and goals for youth, agree that showmanship is a beneficial activity that can develop one's future career skills. The process of showing begins with deciding the type of animal that one would prefer, typically being livestock, such as cattle, pigs, sheep, rabbits, etc. Although some animals are less difficult to handle compared to others, the choice of an animal is based on which one resonates the most with the future shower. The person then spends most of their journey raising and caring for the animal by feeding and grooming them, as well as performing other basic maintenance necessities ("Tomales", n.d.). After the basic care routine, the student gets to demonstrate their showmanship skills - presenting an animal's appearance with a series of poses- at a fair, with the typical goal of selling the animal to receive a profit. With either the same breed or a new breed, the process can then be continually repeated for generations after.

\section{The Aftermath of an Animal}

After showmanship, members exhibit the part of the FFA's motto "Learning to do" by learning to raise an animal for food and then profit. This process can be easy or difficult, depending on the connections that the exhibitor made with their animal (Davonich, 2017). The NPR organization, a nonprofit media organization that provides reliable and trustworthy sources on all topics, differentiates from the FFA by taking into account the emotional aspect of parting from an animal. However, even with this difference, NPR advocates and supports the FFA's motto of "Doing to Learn" and "Earning to Live" as an important way to teach important lessons about the cycle of life and the marketing of an animal to earn a profit. Selling one's animal typically indicates they have been auctioned by potential buyers, including families and businesses, for the result of being slaughtered. Members also can learn animal industry skills by being the ones to butcher the animal they have raised. On the other hand, as all fairs specifically state that animals are not allowed to be taken home after an auction (Bohde, 2019), some members choose to refrain from going to these auctions and decide to keep their animal due to the emotional connections they created. While multiple avenues may be taken after raising an animal, the FFA considers each path as an acceptable way to teach life-long skills. 


\section{Vegetarianism}

People who choose to have a diet consisting of no meat have several influences for their dietary decision, one of which includes their relation to the meat paradox. As cognitive dissonance is the effect of the paradox, individuals must reduce this discomfort through actions that include avoidance, dissociation, and denial. (Rothgerber, 2014) One specific dissonance-reducing strategy is behavioral change, which is when a person adjusts their diet to not include any meat. According to the research on vegetarianism by Rothgerber, a member of the department of psychology, vegetarians "are perceived to have a unique philosophical outlook that includes choices to avoid meat because of a belief system that killing animals for food is unethical" (Rothgerber, 2014). In conjunction, interactions with vegetarians increase dissonance by reminding omnivores of their own discrepancies. While behavioral change may be a preferable strategy, it is typically difficult to change one's diet drastically, so individuals usually choose a different method of practice. Overall, the change to one's diet due to mental incongruity has lasting effects on reducing the perceived cognitive dissonance that takes place when contemplating meat consumption.

\section{Conclusion}

With the five aspects listed, this study will be researching the significance of the FFA's educational program of showing, selling, and working with animals on the cognitive effects of the meat paradox, and whether it leads to more or less dissonance in an individual. The current literature on the link between the meat paradox and FFA, or other animalrelated programs, is lacking, so this paper will investigate if there is a relationship between the two topics. While correlation does not equal causation, a relationship due to the FFA's curriculum could correspond to finding whether the meat paradox is affected when joining a program that works with animals. The significance of this data can determine the FFA's responsibility in being an effective dissonance-reducing strategy, or in contrast, amplifying the inconsistencies within one's brain when thinking about meat consumption. Overall, this data should impact the psychological field in concluding if the FFA, or any activity that works with animals, plays a role in the meat paradox, and determining its either potential helpfulness or increased dissonance in the meat industry.

\section{Method}

This research paper demands an evaluative approach to analyze an influential possibility of the FFA on the paradox contributed by meat. The method of choice would have to be one that compares the two factors of value -the meat paradox in FFA and non-FFA members- to see if the organization may correlate with the group's beliefs. Survey research would, therefore, be the most beneficial to achieve this comparative study, while still achieving a high number of different responses. This method was similarly adapted from a study comparing the meat paradox in China and France (Tian et al., 2015). The survey was distributed to the two countries through a website with anonymity, which was accordingly discussed at a university, where participants then voluntarily decided to complete it. Similarly, the discussion of my survey at school, as well as its anonymity, would allow for many unknown participants to take it, which would ensure a variety of people with secure and undisclosed responses. I would also guarantee privacy through a consent form. Additionally, the French and Chinese meat paradox survey asked questions to both groups about their willingness to eat meat and their perception of cows, allowing the researchers to compare the paradox in the two different countries. While my survey involves more animals as the FFA is a partly animal-based organization, the French and Chinese survey procedures and questions are very beneficial in its ability to compare groups' paradoxical effects. My questions will, therefore, be similar to the example survey but instead be based on FFA and non-FFA members' willingness to eat meat. The survey method will be the most advantageous as it will provide an abundant amount of responses to questions that will allow me to compare the two groups properly. 


\section{Participants}

There is not a specific standard for the qualification of those participating in this survey. Regarding non-FFA participants, their restrictions are evidently nonexistent as they are just representing the average, everyday person. These participants can, therefore, be of any age and gender and engage in any sort of diet. The FFA group, while still having broad requirements, are a little more specific. Obviously, these participants must be a member of the FFA. However, they need to have been a member for at least a year, which will reduce and hopefully eliminate any member who does not take an active participation in the organization and has not learned any curriculum. Both the non-FFA group and FFA group will need at least 50 survey responses each to allow for a range of answers.

\section{Materials}

The materials of this research are minuscule, consisting of at least 100 surveys, as well as consent forms for participants under 18. The surveys, which are necessary in being able to gather the responses, will not ask about the participant's name or gender in order to secure anonymity. Additionally, the survey will ask if the person is a member of the FFA. After that question, the survey will then be used for the thorough examination of one's willingness to eat meat. The first question will be "Are you vegetarian or vegan", along with a question as to why, which will hopefully give the point of view of someone who has had the paradox affect them greatly. There will be five questions, varying depending on the animal of choice, that will all ask the essential question: "Are you willing to eat this animal?" I will have a disclaimer above the questions that says, "If you are willing to eat some part of the animal then answer yes." I will also disclaim that the animals would be considered clean and healthy to eat, as some people might be conflicted about their answer due to those reasons. The five animals that I have chosen to ask about will be a cow, chicken, rabbit, worm, and squirrel.

The basis of using a cow and chicken is just to establish that the person is in fact a meat eater. On the other hand, the rabbit, worm, and squirrel will be the main interest in the survey as they are animals that people in the United States typically do not eat. The person's willingness to eat those animals will provide a better view of the conflicting emotions of eating uncommon animals. After each question, there will be an additional question asking, "If you answered no to the previous question, please answer why." The possible answers to this question will be: "It doesn't/won't taste good," "It feels morally wrong to eat this animal," and "Dietary Reasons." If the person chose that it feels morally wrong, there will be a short answer response at the end of the survey to provide an explanation as to why. These questions will allow me to get an understanding as to why people feel conflicted about eating a specific animal, as well as if being in then FFA correlates to this decision. Along with the surveys, I will need consent forms for the participants that are minors. The consent form will include my name, the research project's title, the purpose of the survey and what it is about, and the risks and potential benefits. I will also ensure that the participant understands that the survey is voluntary, and that confidentiality will be guaranteed through my promise as well as the anonymity of the survey online. Afterward, the minor and their parent will both print and sign their names, as well as include the date of signature.

\section{Procedure}

I will begin this process by creating the anonymous survey online and saving the link. The survey will be discussed in all of my classes, as well as during the FFA club period, in which a teacher or I will then hand out the consent forms for the people who want to participate. Once the consent forms are turned in, a paper with the link to the survey will be handed back to the person. For the participants over 18, they will just be given the link to the survey and will not have to complete a consent form. I will ask my FFA teachers to talk to adults that they know in the organization so they can complete the survey as well. I will retrieve at least 100 responses in total, 50 from each group I am analyzing. 
Once I have gathered all of the responses, I will put the information into an Excel spreadsheet to organize the data efficiently and see trends. I will compare the results from both groups, analyzing the paradox in each, and see if there is a difference between them. If there is a significant difference, I can conclude that the FFA may correlate to answers of specific questions. If there is no difference, then I can conclude that the FFA does not have a correlation with the cognitive dissonance of the meat paradox.

\section{Results}

After my research was completed, a thorough examination of the comparative results between FFA and non-FFA members was made. A total of 151 participants completed the survey, 85 being FFA members, and 66 not being in the FFA. However, six results were discarded for nonsensible answers, such as not eating worms for dietary reasons, leading to 83 FFA members and 62 non-FFA members. The ages ranged from 12 to 59 , with 89 being below the age of 18 and requiring a consent form. With the non-FFA survey responses being a considerably average type of meat consumption, the FFA results showed a distinct dietary change and paradoxical effect in comparison. For an establishment of what is considered "normal" animals to eat in the United States, the results in Figure 1 show no difference between the eating of beef and chicken between the two groups as 137 out of 145 participants were likely to incorporate these meats into their diets.

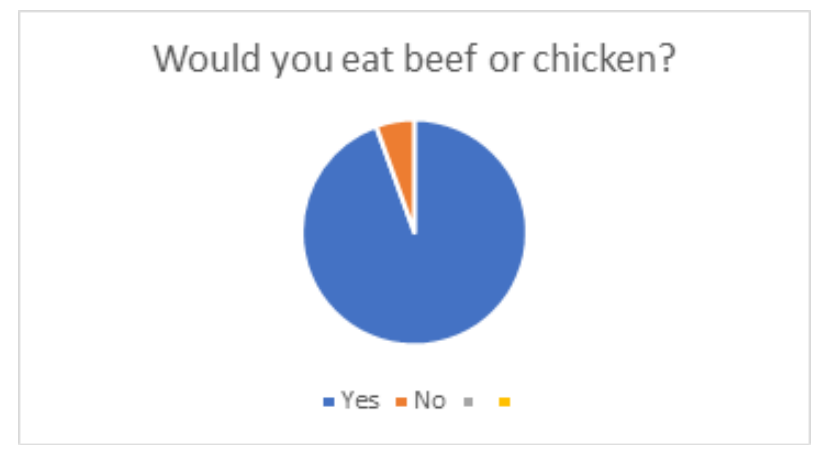

Figure 1: Pie chart displaying all participants' willingness to eat chicken or beef among all participants

The rest of the data showed the contrast between the results of the FFA and non-FFA survey respondents. For instance, the questions that occurred after the beef and chicken-related ones asked about the willingness to eat an animal that could be considered an unusual part of the average American's diet. While these foods may not be incorporated into one's daily lives, the question asked if the individual would at least be willing to try the animal. The first animal that was asked, that may be considered uncommon, was rabbit.

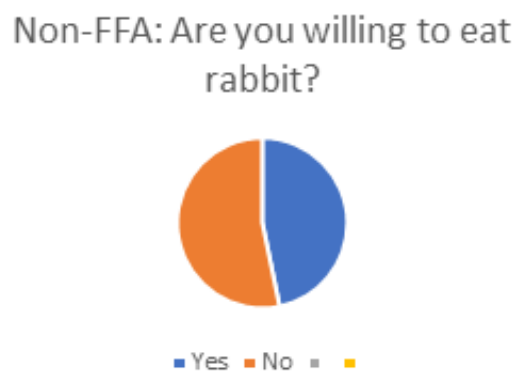

FFA: Are you willing to eat rabbit?

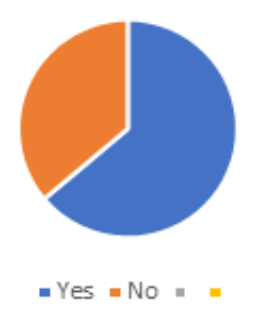

Figures $2 \& 3$ : Pie charts displaying non-FFA and FFA member's willingness to eat rabbit 
As it can be seen in Figures 2 and 3, there is not an extensive connection between one's likelihood to try rabbit and being a member of the FFA. In the non-FFA chart, $46.77 \%$ were willing to eat rabbit, compared to $63.86 \%$ of FFA members. The figures do show more of FFA members who are likely to try rabbit, which can presumably be a result of the curriculum that teaches about the meat rabbit industry. Unfortunately, this small difference is not significant enough to show a relationship between a different cognitive effect associated with eating certain types of meat in the FFA. On the other hand, when comparing the participants' reason to not eat rabbit, the results were distinct. Non-FFA individuals' results show a tie between choosing the option that rabbit would not taste good (40\%), and they would feel morally wrong to eat it $(60 \%)$. By itself, this result does not show any significance in the average consumer's reason to not eat rabbit as the moral and not moral reasons are about equal, with a slight majority choosing the morality option. On the contrary, FFA members display an answer of almost complete agreement about their reason to not eat rabbit. 77\% of FFA members favored the choice that rabbit wouldn't taste good over the choice that eating rabbit would feel morally wrong, resonating with an emotionally unattached response. This result suggests that the FFA typically produces members who have fewer moral attachments to animals, even with working with them. This is probably due to the educational programs that promote raising animals to sell for meat processing.

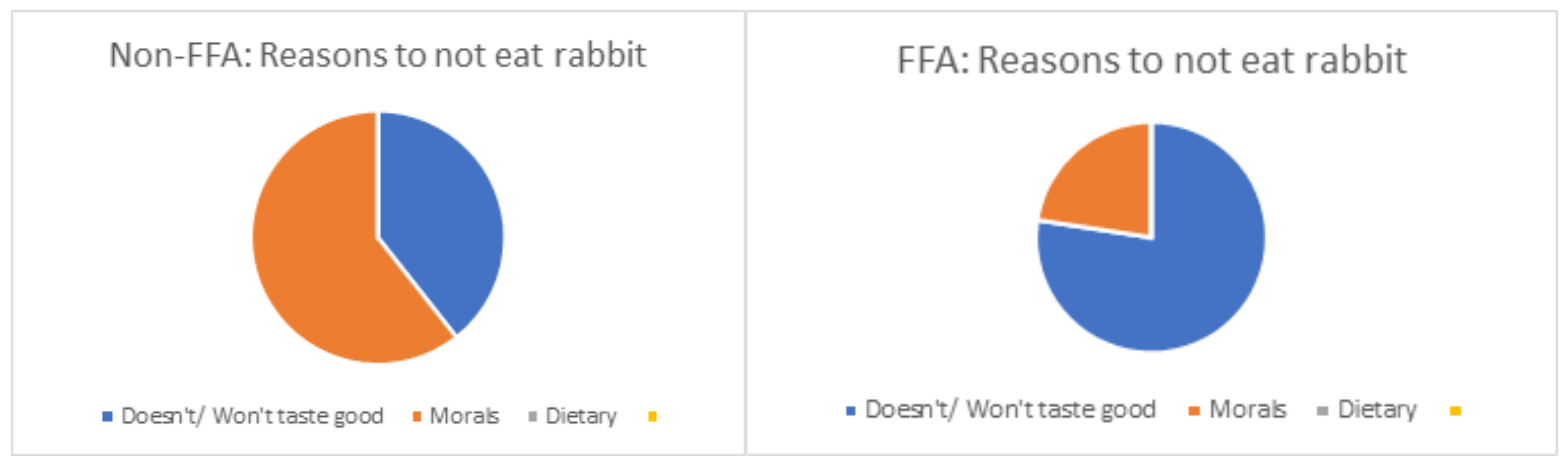

Figures 4 \& 5: Pie charts displaying non-FFA and FFA member's reasons to not eat rabbit

These results can also be seen with squirrels, an animal that is like rabbits in its appearance and overall connotation. The results are different in the willingness to eat the animals but are parallel in the reason to not eat squirrels, as compared to the rabbit pie charts. As seen in Figures 6 and 7,25.81\% of non-FFA members are willing to eat squirrel compared to $51.81 \%$ of FFA members. This is a large discrepancy among the likelihood to eat squirrel compared to rabbits, with a higher amount of people choosing to not eat squirrel. This distinction can be inferred as a different assumption in the safety of eating squirrel. Even with a disclaimer stating that the animals would be safe to eat, squirrels are usually associated with diseases, which can be inferred as to why fewer respondents were willing to eat them. FFA members stayed mostly consistent with their results, decreasing by only $12 \%$ compared to rabbits. They are probably more likely to eat squirrel because they understand the meat industry. They gain knowledge of raising outdoor livestock and eventually processing them for consumption, which allows them to understand the process of making meat safe for consumption more in depth as compared to a non-FFA individual.

Even with that disparity, the charts showing the reasons for participants to not eat squirrel are almost identical to the rabbits' pie charts. Figures 8 and 9 show that non-FFA members had about equal amounts choose the taste and moral option (54\% and 46\%), while FFA members had almost all of the participants choose that they wouldn't eat squirrel due to it not tasting good, more specifically $82.50 \%$. In comparison with the rabbit results, FFA members rarely stated that they would not eat an animal due to moral reasons, leading me to believe that they don't have much emotional attachment to any animal, even with a "cute" connotation. As this result is evident, with more than three quarters of respondents never choosing that they wouldn't eat an animal due to a moral dilemma, it can only lead one to assume that this is due to their curriculum. While there is a possibility of becoming more emotionally attached to animals as one works with them, FFA members actually become unattached. The result may be the effect of either 
learning to not grow emotional attachments to animals as they will end up eventually selling them, the diminished excitement of being exposed to animals over a long period, or just their curriculum. Either way, the FFA has a prominent association of members becoming less attached to an animal of any species, no matter their prolonged time spent with animals.

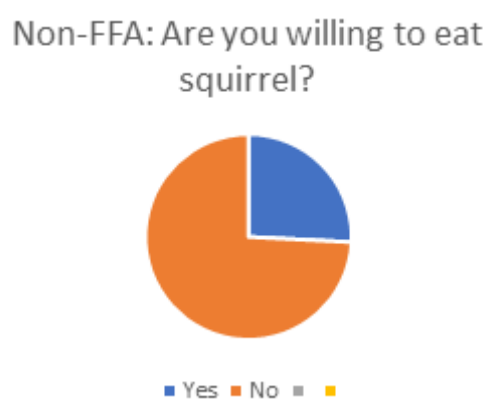

\section{FFA: Are you willing to eat squirrel?}

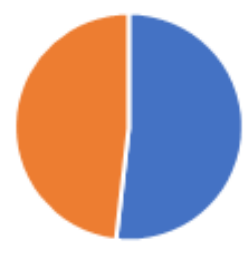

-Yes = No - "

Figures 6 \& 7: Pie charts displaying non-FFA and FFA member's willingness to eat squirrel

Non-FFA: Reasons to not eat squirrel

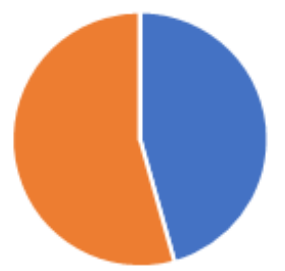

- Doesn't/ Won't taste good | Morak | Dietary |

\section{FFA: Reasons to not eat squirrel}

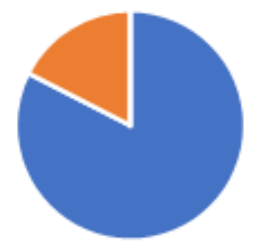

- Doesn't/ Won't taste good - Morak = Dietary "

Figures 8 \& 9: Pie charts displaying non-FFA and FFA member's reasons to not eat squirrel

The last animal that was evaluated was worms. These results contrasted extensively from the other animals by showing data of relative uniformity among all participants in the survey. As can be seen in Figures 10 and 11, 89.40\% of all participants chose that they were unwilling to try worms. In comparison to rabbits and squirrels in which survey partakers would at least try the new variety of food if given the option, almost everyone who took the survey, no matter whether they were a member of the FFA, were unwilling to taste worms. This can assumedly be a result of the negative palatable connotation, with the perception of worms having a peculiar texture and contaminated underground habitat.

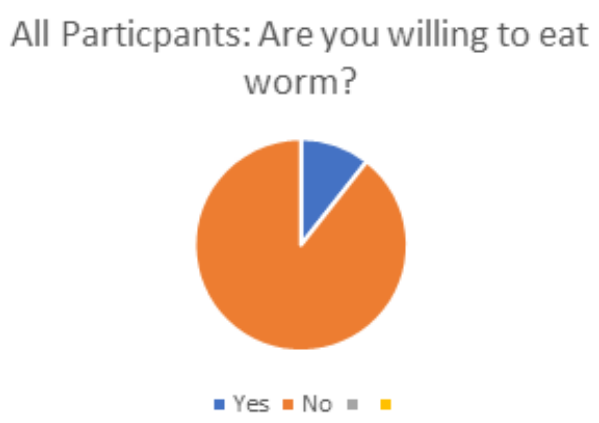

\section{All Participants: Reasons to not eat worms}

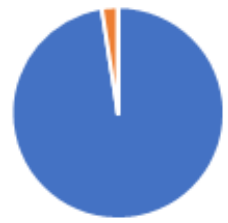

- Doesn't tastegood | Morak | Dietary |

Figures 10 and 11: Pie charts displaying all participants' willingness to eat worms and the reasons why 
Accordingly, another consistency between participants occurred when asked the reason to not partake in the consumption of worms. $92.97 \%$ of respondents chose that they would not eat worms because they don't believe it would taste good. Almost no one had a moral connection to the animal. Participants then attempted to explain their morality choices with sentences that were consistent with "I only feel bad eating squirrels and rabbits because they're cute." Their explanation as to why they feel bad about eating rabbit and squirrel, rather than worms, remain consistent with the results in Figure 11, as well as the basic principles of the meat paradox.

The moral connections people have with certain animals are mainly based on perception. As rabbits and squirrels have a positive stigma, people create emotional attachments to the species when reflecting on their willingness to eat them. Worms have a negative, or indifferent, perception, hindering the creation of moral relationships with them. Cows and chicken, on the other hand, are taught to be perceived as "normal" animals to eat, as participants consistently said in the open response question, "Cows and chickens are grown and maintained to eat." This instilled belief almost completely prevents anyone from feeling strong emotions that make them unwilling to eat the farm animal. The farm animal results ended up producing primary examples of the meat paradox in all Americans, no matter the club one's affiliated to. However, the rest of the data suggests that the FFA corresponds to members' reduced personal relationships and moral attachments to uncommonly eaten mammalian species.

\section{Conclusion}

This data, conclusively, suggests an important correlation between the morality of individuals and the membership in the FFA. However, even with these distinguished results, there were some limitations with this research study, one most importantly being that correlation does not equate to causation. While my results do suggest a possible relationship between the FFA and cognitive dissonance, it cannot be certain whether the FFA was the cause of this change in morality. There may be an unknown cause of FFA members' morals that I did not explore. Either way, my results did not allow for an understanding of any sort of cause, it only educated me on a notable trend.

Another important limitation of my study was the possibility of bias or a hidden agenda. Like most survey research, I am unable to prevent my participants from answering dishonestly. There is also the probability of acquiescence bias, which is when the respondent agrees with whatever I am asking. As my survey consisted of answer choices surrounded by the idea of morals, the respondents might have felt it easier to agree with my attempted hidden implications. On the other hand, the participants could have realized the implications and gave effort into disproving what was being implied. Either way, there is a possibility of dishonesty in my survey, so the results may not be completely accurate. However, even with this possibility, there was a distinguished trend among FFA and non-FFA members, which shows a high level of accuracy among most of the answers.

In addition to the list of limitations of survey research, there may have been a difference in the interpretation of the questions. Even with my disclaimers, people may have been confused about whether I was asking them if they would try the food or if they would continue to eat the food in their daily lives. When I asked about their willingness to eat an animal, I was asking were they willing to try it once, if offered. Obviously, if they are not being forced to eat the food, people may be unwilling to try it once as there are a variety of other meats to eat. This could have influenced some answers in a negative way. Even with multiple established disclaimers, I am unfortunately unable to avoid all misinterpretations of questions and answers.

Although there are limitations to conducting survey research, my results still display a level of accuracy and importance that can be incorporated into the current body of research of the meat paradox and cognition. In a different research essay, the study was conducted to find a solution to the paradox and additional ways to maintain it (Bastian \& Loughnan, 2016). My study can be a helpful source that can be integrated in their continued investigation of the maintenance of the paradox. The researchers of this study may find my results indicative of increased interactions between humans and animals to be a possible solution to the meat paradox. As people interact and work with animals on a daily basis, they tend to feel fewer moral connections to most animals in general, which can be a solution to reducing the paradox and allowing people to eat a greater variety of meat as well. 
Furthermore, the study on the solution of the meat paradox found that their study could be implicated into research on dehumanization, which is reducing the perceived consequences of harmful acts towards others (Viki et al., 2013). My research, too, can be helpful in the understanding of unethical behaviors towards humans. The understanding of the reduced moral capabilities towards animals when one is closely associated to them can be introduced into other psychological research on the killings of people. As animals can be objectified as creatures that are meant to be killed for food instead of as living beings, individuals can also be viewed as less-than-human, which can be justifications to facilitate death.

The results of this study show that future research on this subject must account for other variables in order to encapsulate a complete understanding of the interactions between people and animals, along with the incorporation of the FFA, as influential to the meat paradox. I never asked about the gender of my participants so further research should be completed to reveal if there is a distinction among them in the FFA. While there is already present research of the meat paradox between two genders, the association of the FFA, or other activities associated with increase animal and human interaction, might cause different results among genders. Additionally, more research on the different fields of the FFA in relation to the paradox needs to be conducted. Being in the veterinarian field compared to the agricultural field may produce different results among respondents. I cannot be sure if I received more participants in one field of the FFA, which may have swayed my results if the fields have different beliefs.

Overall, my results had a definite conclusion that there's a relationship between being in the FFA and a lesser moral connection to animals, as few FFA members chose that they had any moral connection to an animal when they weren't willing to eat it. The other conclusive result of my data is that there are differing moral connections to animals with particular stigmas, as can be seen with worms, an animal with a negative appeal. Altogether, this study can be beneficial in current and future bodies of research on the topic of the meat paradox and will hopefully forever reshape the perceived moral capabilities of the animal and human relationship in psychology.

\section{Acknowledgments}

I would like to thank my teacher, Mrs. Poole, for guiding me through this academic journey to explore and understand an interesting topic. I would also like to thank my mom for providing constant support throughout this past year while I studied this new topic.

\section{References}

Rozin, P. (1996). Towards a psychology of food and eating: From motivation to module to model to marker, morality, meaning, and metaphor. Current Directions in Psychological Science, 5(1), 18-24. https://doi.org/10.1111/1467$\underline{8721 . e p 10772690}$

Loughnan, S., Haslam, N., Bastian, B. (2010). The role of meat consumption in the denial of moral status and mind to meat animals. Appetite, 55(1), 156-159. https://doi.org/10.1016/j.appet.2010.05.043

Loughnan, S., Bastian, B., Haslam, N. (2014). The psychology of eating animals. SAGE Journals, 23(2), 104-108. doi: $10.1177 / 0963721414525781$

Buttlar, B. \& Walther, E. (2018). Measuring the meat paradox: How ambivalence towards meat influences moral disengagement. Appetite, 128, 152-158. https://doi.org/10.1016/j.appet.2018.06.011

FFA. (n.d.). Retrieved April 1, 2020, from https://www.ffa.org/ 
FFA Vision, Mission and Motto. (n.d.). Retrieved April 1, 2020, from https://www.ffa.org/about/who-we-are/mis$\underline{\text { sion-motto/ }}$

SAE for ALL Evolving the Essentials. (n.d.). Retrieved April 3, 2020, from https://saeforall.org/immersionsae/?wizard

Tomales FFA. (n.d.). Retrieved April 3, 2020, from https://www.tomalesfriendsofagriculture.org/tomales-ffa.html

Danovich, T. (2017, August 30). For 4-H Kids, Saying Goodbye To An Animal Can Be The Hardest Lesson. Retrieved April 3, 2020, from https://choice.npr.org/index.html?origin=https://www.npr.org/sections/thesalt/2017/08/30/545603450/for-4-h-kids-saying-goodbye-to-an-animal-can-be-the-hardest-lesson

Bohde, K. J. (2019, November 21). 4-H General Livestock Rules. Retrieved April 3, 2020, from https://extension.purdue.edu/hamilton/article/4406

Rothgerber, H. (2014). Efforts to overcome vegetarian-induced dissonance among meat eaters. Appetite, 79, 32-41. https://doi.org/10.1016/j.appet.2014.04.003

Tian, Q., Becker, M., Hilton, D. (2015). Confronting the meat paradox in different cultural contexts: Reactions among Chinese and French participants. Appetite, 96, 187-194. https://doi.org/10.1016/j.appet.2015.09.009

Bastian, B., Loughnan, S. (2016). Resolving the Meat-Paradox: A Motivational Account of Morally Troublesome Behavior and Its Maintenance. Personality and Social Psychology Review, 21(3), 1-22. doi: $10.1177 / 1088868316647562$

Viki, G. T., Osgood, D., \& Phillips, S. (2013). Dehumanization and self-reported proclivity to torture prisoners of war. Journal of Experimental Social Psychology, 49(3), 325-328. https://doi.org/10.1016/j.jesp.2012.11.006 